\title{
MODOS DE INTEGRACIÓN DE LABORATORIOS ONLINE EN SISTEMAS DE GESTIÓN DE APRENDIZAJE
}

\author{
Ildefonso Ruano Ruano \\ alonso@ujaen.es \\ Javier Gámez García \\ jggarcia@ujaen.es \\ Juan Gómez Ortega \\ juango@ujaen.es
}

\begin{abstract}
Resumen
El trabajo práctico que se desarrolla en los diferentes tipos de laboratorios es esencial para la obtención de una formación completa en los estudios de ingeniería. La aplicación de las Tecnologías de la Información y las Comunicaciones en la educación ha traído consigo muchos cambios en la forma de impartir la docencia, se puede decir que el mayor exponente de estos cambios lo constituye el uso de los Sistemas de Gestión de Aprendizaje, más conocido por LMSs o Learning Management System, en inglés. Estos cambios también se han visto reflejados en las prácticas de laboratorio con el uso de los laboratorios online. Este trabajo analiza las posibilidades de integración que pueden darse entre los laboratorios online y los LMSs, establece una clasificación de modos de integración, indica los estándares más utilizados para lograr esta integración cuando el modo así lo requiere y las características que presentan incidiendo en los beneficios que se pueden obtener en cada uno de los modos. También se muestra el modelo seguido en la Universidad de Jaén que ha sido aplicado en asignaturas de Grados de Ingeniería Industrial, Ingeniería Telemática e Ingeniería de Telecomunicaciones.
\end{abstract}

Palabras Clave: Educación, Laboratorios online, Sistemas de Gestión de Aprendizaje.

\section{INTRODUCCIÓN}

La importancia del trabajo práctico en la formación universitaria de los estudiantes de ingeniería es un hecho indiscutible que viene contemplado en todos los planes de estudio [9] [10]. Tradicionalmente, este tipo de formación se ha realizado en sesiones presenciales que se realizan en espacios diseñados especialmente para ello (laboratorios) o en entornos reales (visitas a centros especializados o trabajos de campo en exterior). En cualquiera de estas modalidades, la formación práctica suele requerir un coste importante. Cuando se trata de un laboratorio docente es necesario reservar en exclusiva una dependencia, un coste inicial de recursos muy importante y de mantenimiento de los mismos, el cual suele incluir a personal especializado. Cuando se trata de trabajo en entornos reales también se tiene un coste importante, al menos el asociado a los medios de transporte y personal de apoyo. La formación práctica tradicional presenta, además, bastante restricciones en los dominios espacio-temporales; este tipo de trabajo práctico requiere estar en un sitio y momento determinado. Por estos motivos no es de extrañar que se aplicaran las Tecnologías de la Información y las Comunicaciones (TIC) a los laboratorios tratando de solventar estos problemas. De este modo surgieron los laboratorios online, que permiten el acceso de los estudiantes a trabajos prácticos a través de Internet eliminando las restricciones espacio-temporales y reducir el coste de desarrollo y mantenimiento de los mismos. Además presentan otra serie de ventajas como son facilitar el uso compartido entre distintas instituciones, ofrecer mayor seguridad a los usuarios y extender el uso de recursos escasos. Cuando los estudiantes trabajan con sistemas reales remotos a través de Internet se dice que se tiene un laboratorio remoto (RL o Remote Lab en inglés) mientras que cuando el estudiante trabaja con una simulación de un sistema real a través de Internet se dice que se trata de un laboratorio virtual (VL o Virtual Lab en inglés). Finalmente, cuando en un mismo laboratorio se trabaja con recursos reales y simulados se dice que es un laboratorio híbrido. En cualquiera de sus modalidades los laboratorios online o VRL (Virtual/Remote Lab) [25] pueden considerarse como contenidos de enseñanza electrónica a través de Internet, más conocido como e-learning.

Sin embargo, hoy en día el mayor exponente de la aplicación de las TIC en la educación superior viene dado por el uso de los Sistemas de Gestión de Aprendizaje, Learning Management System en inglés, más conocido por las siglas LMS [24]. No existe ninguna organización universitaria que no haga uso de un LMS. Los LMSs pueden servir como apoyo para impartir docencia la docencia presencial, 
para impartir docencia totalmente virtual a través de Internet (e-learning) o en forma mixta (en inglés blended learning o b-learning) [20].

Era lógico que se produjera una convergencia entre los laboratorios online y los LMS, esta idea ya fue introducida por algunos autores [15] y desarrollada de múltiples formas [6], [23], [33], [18]. En este artículo se analiza esta integración, se identifican los distintos modos en los que se puede encontrar, se exponen las posibilidades técnicas de implementar cada modo, las ventajas e inconvenientes que presenta cada uno de ellos y finalmente se explica como los autores de este trabajo están realizando los laboratorios online en la Universidad de Jaén (UJA) como ejemplo de implementación de uno de los modos de integración mostrados.

El resto del trabajo está estructurado de la siguiente forma: en el apartado 2 se tratan los LMSs mostrando las ventajas y características que suelen presentar. El apartado 3 presenta un breve estado del arte de la integración de laboratorios on-line en los LMSs. El apartado 4 muestra una clasificación de los niveles de integración Lab-LMS que pueden darse analizando las ventajas que se obtienen en cada uno de ellos y contrasta modelos de integración basados en estándares que se han usado o se pueden usar para facilitar la integración Lab-LMS mientras que en el apartado 5 se presenta con más detalle un modelo de integración basado en los estándares SCORM (Shared Content Object Reference) [5], seguido en la UJA. Para finalizar, en el apartado 6 se muestran las conclusiones que se han obtenido de este trabajo.

\section{LMS: SISTEMAS DE GESTIÓN DE APRENDIZAJE}

Trend es una herramienta de la empresa Google que permite representar la frecuencia con la que se ha buscado un término en Internet en porcentaje (considerando $100 \%$ el momento en el que la frecuencia de la búsqueda fue mayor). La figura 1 muestra los resultados obtenidos para el término LMS, estos datos confirman que los Sistemas de Gestión de Aprendizaje están en auge y en su mayor nivel de interés. Recientes estudios confirman esta idea y añaden que el ámbito de la integración LabLMS también es un asunto en auge [17].

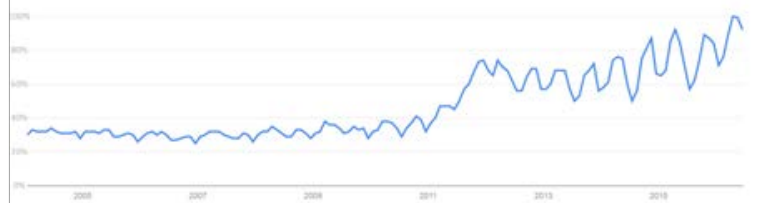

Figura 1: Resultado global de la tendencia de búsqueda del termino LMS en Internet.

Existen muchos LMS, más de 340 [7], atendiendo al desarrollo y propiedad del software asociado se pueden establecer tres tipos de LMS: comerciales (Blackboard, Edoceo, Desire2learn, etc.), de software libre (Moodle, ILIAS, Dokeos, Sakai, etc.) y de desarrollo propio (Ágora virtual, EducaLab, Virtaula, etc.). Los más utilizados en las universidades de España son los de software libre, y especialmente Moodle, implantado en más de 35 universidades [27]. Un LMS es una herramienta esencial en la educación universitaria una vez realizada la identificación de un usuario, la cual puede realizarse de forma federada, puede proporcionar las siguientes ventajas:

- Ofrecen bajo un mismo entorno web espacios virtuales que permiten integrar la oferta educativa de los organismos de educación superior.

- Permiten ampliar la oferta educativa y diversificarla.

- Ofrece posibilidades de intercambio y exposición de contenidos docentes.

- Permiten establecer secuencias de aprendizaje.

- Ofrece posibilidades de comunicación y colaboración entre usuarios.

- Ofrece posibilidades de evaluación a los usuarios.

- Permiten realizar el seguimiento del trabajo realizado por los usuarios.

- Ofrecen soporte multilenguaje.

Para ello suelen ofrecer los siguientes recursos y servicios:

- Espacio Virtual personalizado, con posibilidad de almacenamiento online de contenidos privados y logros obtenidos (informes, badges, etc.).

- Gestión de usuarios, incluyendo su pertenencia a grupos y permisos sobre los recursos.

- Recursos de aprendizaje en diferentes formatos (propios, pdf, docx, etc.) como pueden ser módulos de teoría.

- Herramientas de comunicación como foros, chats, sesiones de videoconferencia, etc.

- Herramientas de creación de contenidos con posibilidad de permitir trabajo colaborativo como páginas web, wikis, módulos, blogs, portafolios, documentos en distintos formatos, etc.

- Herramientas de recogida de opiniones de usuarios como encuestas y/o votaciones.

- Herramientas de evaluación de usuarios como tests, tareas y trabajos on-line así como de la gestión de los mismos, los resultados y las comunicaciones a los alumnos.

- Sistema de seguimiento del progreso de aprendizaje de los usuarios basados en números de accesos, tiempo de trabajo, 
competencias, objetivos y superación de eventos.

Unas de las características más valoradas en los LMS son la estandarización y la interactividad, las cuales permiten reutilizar contenidos en distintos sistemas y el intercambio de información entre un LMS y otro sistema. Estas propiedades son muy importantes a la hora de considerar la integración de un LMS con un laboratorio online ya que permiten ampliar las posibilidades de llevarlo a cabo. En este sentido conviene destacar que existen múltiples estándares relacionados con el e-learning, esta estandarización no se ha centrado sólo en la organización $\mathrm{y}$ descripción de los contenidos (resuelven el problema de la interoperabilidad y portabilidad) sino también en aspectos dinámicos que permiten describir procesos educativos complejos que permitan crear aplicaciones más complejas, por ejemplo que sean colaborativas o estén basadas en competencias. En [19] se muestran diferentes categorías que pueden ser objeto de una estandarización: Accesibilidad, Arquitectura, Calidad, Competencias, Contenidos y Evaluación, Derechos digitales, Información del alumno, Interactividad, Metadatos, Vocabulario y Lenguajes, Proceso de Aprendizaje y Repositorios. Los estándares más estrechamente relacionados con la integración Lab-LMS son los catalogados como "Contenidos y Evaluación" e "Interactividad". Ejemplos de los primeros son la sub-especificación SCORM CAM (Content Aggregation Model) [2] o IMS QTI (Instructional Management System, Question and Test Interoperability) [21], tratan patrones de creación de contenidos con posibilidad de empaquetado y su evaluación, y como ejemplos de los segundos están la sub-especificación SCORM RTE (Run Time Environment) [3], LTI (Learning Tool interoperability) [22] o xAPI (Experience API, también conocido como Tin Can API o TCAPI) [34], que tratan el intercambio de información entre recursos de aprendizaje y LMS durante la ejecución de los mismos.

\section{ESTADO DEL ARTE}

Los primeros trabajos científicos que trataron el tema de los laboratorios online fueron investigaciones pioneras que se desarrollaron centrándose más en los problemas técnicos particulares que requería la puesta en marcha de los mismos. Estos laboratorios se ejecutaban de forma totalmente independiente a los LMS. Varios autores apuntaron la idea de diseñar laboratorios online sin olvidar aspectos pedagógicos, pensando en la efectividad del aprendizaje [11], y otros señalaron la posibilidad de cerrar el hueco que había entre los laboratorios online y los LMS [15]. El primer acercamiento se produjo cuando empezaron a desarrollarse redes de laboratorios online que utilizaban plataformas Web especializadas (por ejemplo eMersion [14]) que permitían el acceso y compartición entre distintos centros y organismos (proyecto AutomatLabs [39], proyecto RCL [16], proyecto Lila [12], proyecto iLab [40], DeustoLabs [13]). La mayoría de estas plataformas permiten la compartición de laboratorios remotos (algunos también virtuales) mediante una determinada tecnología como pueden ser los Web Services para conectarse a los equipos remotos que realizan el control directo de los recursos reales, sin embargo operaban de forma paralela a los LMS institucionales sin la posibilidad de aprovechar las ventajas pedagógicas que esto supone $\mathrm{y}$, por lo tanto, sin permitir interacciones entre el laboratorio y el LMS. Algunas de estas redes de laboratorios online evolucionaron para integrarse con LMSs (por ejemplo, a partir de AutomatLabs surgió UNILabs). Posteriormente, siguiendo esta línea de trabajo se han realizado múltiples trabajos aportando diferentes soluciones [1] [35]. La integración Lab-LMS se ha acometido siguiendo ideas tan variadas como la de usar los LMS como simples contenedores de los laboratorios online, pero sin establecer comunicaciones entre el software del laboratorio y el LMS en el que estaban alojados. Una segunda evolución en la integración Lab-LMS se dio cuando se empezaron a desarrollar laboratorios que eran capaces de comunicarse con el LMS, en este sentido se han realizado propuestas basadas en el desarrollo de software específico para la adaptación de los laboratorios en un determinado LMS [8] [38] y otras basadas en el uso de estándares que permiten la reutilización del laboratorio en los LMS compatibles [33]. Esta última posibilidad puede implementarse de dos formas diferentes que se diferencian en la ubicación del laboratorio; alojado en el mismo LMS [29] o ejecutándose de forma totalmente independiente al LMS aunque utilizando estándares de comunicación con el LMS que permiten su integración en el mismo [26]. En [37] se propone LaaS (Laboratory as a Service), un modelo de desarrollo de laboratorios remotos como componentes modulares con capacidad de interactuar con sistemas remotos, lo cual permite tanto la comunicación con el recurso remoto como su integración con LMSs.

\section{INTEGRACIÓN LAB-LMS}

La integración Lab-LMS puede proporcionar diferentes beneficios dependiendo del modo en que ésta se produzca. Antes de analizar los modos de integración y beneficios que se pueden obtener es importante tener en cuenta las siguientes ideas:

a) Un LMS es el elemento tecnológico central de la vida docente universitaria, en él los alumnos encuentran los recursos y herramientas necesarias para realizar sus tareas estudiantiles y los profesores y tutores sus labores docentes. 
b) Un laboratorio online implica la existencia de una aplicación o software de laboratorio que a partir de este momento se va a llamar VRL. El VRL debe presentar siempre una interfaz gráfica de usuario (GUI en inglés) a través de la cual el usuario interactúa con un sistema real, en caso de tratarse de un VL, o con una simulación que debe estar incluida en el mismo, en caso de tratarse de un VL (si fuera un laboratorio híbrido la GUI se usaría para interactuar con un sistema real y una simulación simultáneamente).

c) El VRL es un contenido de aprendizaje que, desde el punto de vista pedagógico, no debe presentarse de forma aislada a los alumnos. Al VRL le deben acompañar otros recursos que lo apoyen y sirvan para mejorar la efectividad del aprendizaje que se pretende obtener, sólo por señalar algunos se puede mencionar los siguientes: Lista de requisitos técnicos para ejecutar el VRL, Manual de uso del VRL, Información sobre el laboratorio online y los objetivos y/o competencias que se pretenden obtener, Contenidos teóricos de la materia relacionada, Guiones de las prácticas que deben realizarse, Pruebas de evaluación, ...

Se ha establecido una clasificación de modos de integración de un VRL con un LMS. La tabla 1 muestra un resumen de las principales características que presentan cada uno de los modos. El modo 0 equivale a un modo sin integración y según aumenta el número aumenta el grado desde un nivel de integración mínima (1) a superior que puede establecerse de 3 modos diferentes (3, 4 o 5).

0 . No integración. El LMS y el VRL no establecen ninguna relación, ni siquiera de enlace de uno al otro ni por supuesto con ningún otro contenido del LMS. No existe ningún beneficio al no existir ningún tipo de integración.

1. VRL enlazado en LMS sin comunicaciones. En el espacio virtual de la asignatura o materia de la que forma parte el VRL, que está en el LMS, existe un enlace al VRL, el cual se encuentra ubicado en otro servidor.
Esta integración es mínima, el único beneficio que se aporta es la facilidad de encontrar el VRL a través del LMS, que es un elemento habitual de uso.

2. LMS como contenedor del VRL. El VRL se encuentra ubicado en el LMS, en el espacio virtual de la asignatura o materia junto al resto de recursos de la misma. Esta integración es algo mayor, el beneficio está claro, por un lado se asegura que la ejecución del VRL sólo se produce por usuarios identificados por el LMS. Por otro lado el alumno encuentra todos los recursos en el mismo entorno sin necesidad de acceder a otras direcciones en Internet. Además, si el LMS lo permite, algo que suele ser habitual, se pueden establecer secuencias o caminos de aprendizaje para controlar el acceso al VRL basado en la superación de otro u otros recursos (control de secuencia Pre-VRL). Un ejemplo de este tipo de integración es el que se da cuando se incrusta un VRL como parte de contenido SCORM pero sin utilizar la subespecificación RTE para establecer comunicaciones con el LMS que lo aloja.

La figura 2 muestra esquemas de los tres primeros modos de integración (modos 0 a 2).

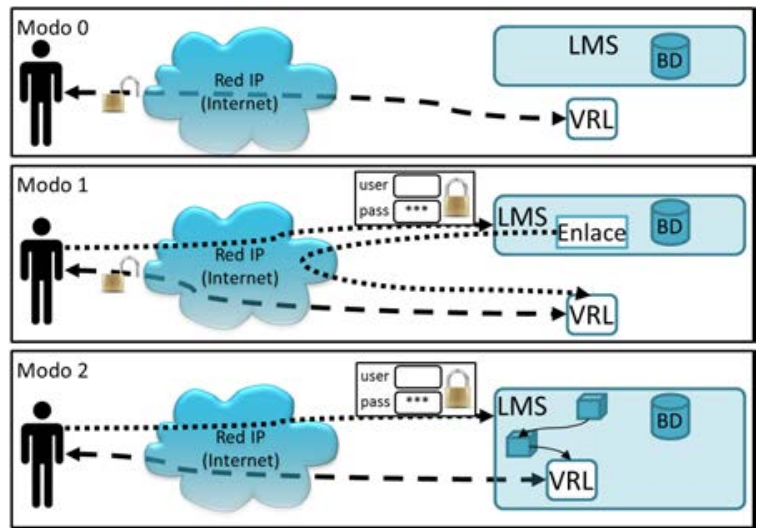

Figura 2. Esquemas de modos de integración 0 a 2.

3. LMS como contenedor del VRL que interacciona con el LMS mediante un

\begin{tabular}{|c|c|c|c|c|c|c|c|c|}
\hline \multirow{2}{*}{ Integración } & \multirow{2}{*}{ Modo } & \multirow{2}{*}{ Ubicación } & \multirow{2}{*}{$\begin{array}{c}\text { Comunicación } \\
\text { con LMS }\end{array}$} & \multicolumn{2}{|l|}{ Rect } & \multirow{2}{*}{ Identificación } & \multirow{2}{*}{ Portabilidad } & \multirow{2}{*}{ Personaliza } \\
\hline & & & & Pre-VRL & Post-VRL & & & \\
\hline Nula & 0 & Ext & No & No & No & No & Sí & No \\
\hline Mínima & 1 & Ext. (enlace) & No & No & No & $\mathrm{No}^{1}$ & No & No \\
\hline Media & 2 & LMS & No & Posible & No & LMS & No & No \\
\hline \multirow{3}{*}{ Superior } & 3 & LMS & Estándar & Posible & Posible & LMS & Posible & Posible \\
\hline & 4 & LMS & Propia & Posible & Posible & LMS & $\mathrm{No}^{2}$ & Posible \\
\hline & 5 & Ext. y LMS ${ }^{3}$ & Estándar & Posible $^{3}$ & Posible $^{3}$ & VRL-LMS & Posible & Posible \\
\hline
\end{tabular}

Tabla 1: Modos de Integración Lab-LMS y características de cada uno.

${ }_{1}$ Aunque el enlace se encuentre únicamente en el LMS no se puede asegurar el control de acceso al VRL.

${ }^{2}$ Cuando se hace uso de estándares la portabilidad entre LMSs compatibles está asegurada, al usar desarrollos propios no.

${ }^{3}$ Si el LMS implementa un recurso para el estándar y permite establecer control de acceso con otros recursos asociados. 
estándar de comunicaciones. Este caso es el mismo que el anterior pero se ha añadido la posibilidad de que el VRL y el LMS se comuniquen. $\mathrm{Si}$ el LMS transmite la identificación del alumno al VRL, le permitiría realizar un aprendizaje adaptado al mismo y, una vez que el alumno termine de trabajar realizando los experimentos, comunicar la calificación y su resultado al LMS. De este modo se podría usar este resultado en el LMS para controlar el acceso a otros recursos asociados incluidos en el LMS (control de acceso a recursos asociados Post-VRL). Al usarse estándares de comunicación se podría exportar el VRL para poder usarse en otros LMS que soporten el mismo estándar. Un ejemplo de este tipo de integración es el que se da cuando se incrusta un VRL como parte de contenido SCORM que utiliza la subespecificación RTE para establecer comunicaciones con el LMS que lo aloja, en este caso la comunicación está limitada al intercambio de información que pertenezcan al modelo de datos RTE de SCORM. También se podría realizar utilizando el estándar xAPI, aunque en este caso es necesario que se utilice un LRS (Learning Record Store) que puede estar implementado en el mismo LMS para que se haga cargo del almacenamiento de los registros de la actividad de aprendizaje generados por el VRL y establecer un "vocabulario" que permita definir los datos que se quieren intercambiar entre el VRL y el LMS.

4. LMS como contenedor del VRL que interacciona con el LMS mediante el desarrollo de una extensión del LMS. Este modo sería muy similar al anterior salvo que en vez de usar un estándar para establecer las comunicaciones VRL-LMS se usaría una extensión que se debe añadir al LMS para que sea capaz de comunicarse con los VRL que sean creados de un modo específico. Presenta el problema de que los VRL sólo pueden ser reutilizado en otros LMSs que sean del mismo tipo y se les haya instalado la misma extensión.

5. VRL externo al LMS que interacciona con el LMS mediante un estándar de comunicaciones. En este modo el LMS se ejecuta de forma totalmente externa al LMS, puede hacerlo en cualquier dispositivo para el que se haya programado el VRL (móvil, Tablet, PC, etc.). El VRL debe recoger las credenciales del usuario y usar un estándar de comunicaciones que soporte el LMS (la identificación podría realizarla el LMS con las credenciales transmitidas el VRL), habrá que configurar en el LMS un objeto relacionado con el estándar en el que se configurará los parámetros de la comunicación que se va a realizar con el VRL. Entre estos parámetros es necesario utilizar un "vocabulario" que permita la comunicación VRL-LMS. Si el LMS lo permite, este objeto podría relacionarse con los otros objetos relacionados con el VRL que hay en el LMS estableciendo secuencias de aprendizaje Pre-VRL y Post-VRL. Además, el VRL sería reutilizable en todos los LMS que cumplieran el estándar utilizado por el VRL. Al igual que los modos 3 y 4 podría permitir un aprendizaje personalizado. Un ejemplo de este tipo de integración es el que se da cuando se tiene un VRL que utiliza el estándar LTI para establecer comunicaciones con el LMS que lo aloja. También se podría utilizar el estándar xAPI que se comentó en el modo 3, ya que no es necesario que el contenido se encuentre en el mismo LMS.

La figura 3 muestra esquemas de los modos de integración 3 a 5 .

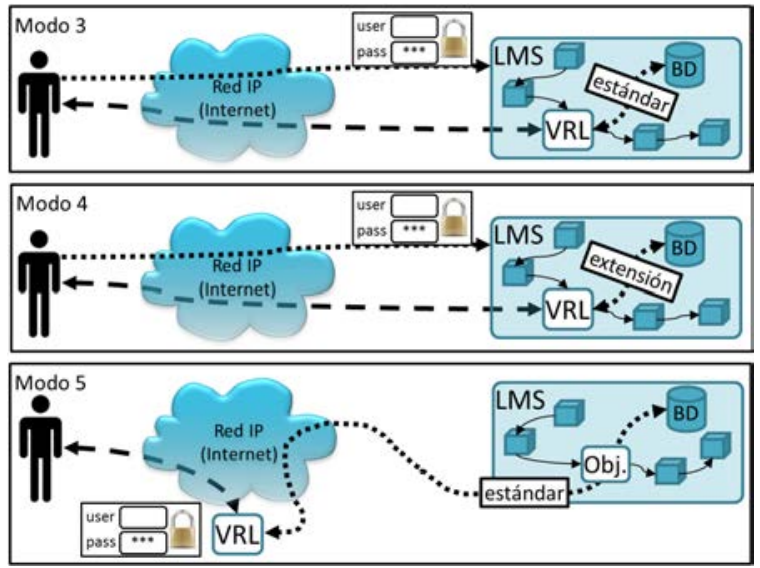

Figura 3. Esquemas de modos de integración 3 a 5 .

La personalización del aprendizaje o funcionamiento adaptado al estudiante que pueden ofrecer los VRL en los modos 3 a 5 debe programarse en el propio VRL. Cuando esta personalización se quiera reflejar en el LMS deberá tenerse en cuenta el vocabulario que tenga en cada caso particular, el cual puede depender del estándar o extensión utilizada. Entre las opciones de personalización que se podrían dar estarían las siguientes:

- Personalización de los experimentos a realizar, cada alumno realizaría un experimento diferente en función de su identificación.

- Posibilidad de almacenar notas personales y/o resultados del trabajo realizado en el 
LMS para su recuperación en ejecuciones futuras.

- Posibilidad de personalizar el funcionamiento del VRL, por ejemplo permitir la selección de lenguaje, rapidez de ejecución, volumen, aparición de subtítulos y de almacenar estas preferencias en el LMS para ejecuciones posteriores.

- Posibilidad de personalizar la evaluación de las prácticas y almacenar dichos valores en el LMS. No existe ninguna definición oficial de lo que es una integración avanzada entre un laboratorio online y un LMS, pero se puede decir que ésta se produce cuando existe una comunicación entre ambos que permite cumplir al menos esta propiedad.

Todas ellas son características deseables desde un punto de vista pedagógico ya que permiten elaborar contenidos docentes de mayor calidad.

\section{INTEGRACIÓN LAB-LMS CON SCORM EN LA UJA}

En la UJA, tras la experiencia obtenida, los autores de este trabajo han elaborado una metodología que se está utilizando actualmente para crear los laboratorios online [30]. Esta metodología parte de los objetivos docentes que se quieren conseguir y acaba con la obtención de un laboratorio online integrado en LMS y efectivo desde el punto de vista del aprendizaje del alumno. La metodología es genérica y aunque propone que exista una integración Lab-LMS no obliga a realizarse por ningún modo en especial. SCORM es el estándar elegido como soporte de laboratorios online integrados en modo 3 con ILIAS, el LMS institucional. Estos laboratorios se están presentando a alumnos de grados de Ingeniería Industrial, Ingeniería Telemática e Ingeniería de Telecomunicación en las Escuela Politécnica Superior de Jaén y la Escuela Politécnica Superior de Linares.

El conjunto de estándares SCORM incluye 3 subespecificaciones que son utilizados para la definición de contenidos de e-learning. Hoy en día, y a pesar del tiempo que ha pasado desde su publicación, continúa siendo el estándar específico de creación de contenidos de e-learning más extendido y soportado por los LMS del mercado. La sub-especificación CAM [2] permite definir una estructura de contenidos de aprendizaje basado en páginas Web y los elementos incluidos en dichas páginas, los cuales se empaquetan en un fichero de formato ZIP llamado paquete SCORM. La sub-especificación SN (Sequencing and Navigation) [4] permite establecer la forma en la que se puede navegar entre los objetos $\mathrm{y}$ recursos incluidos en el paquete SCORM. Finalmente la sub-especificación RTE [3] define la forma en que el LMS debe ofrecer los contenidos del paquete SCORM y la forma de comunicación que se puede establecer entre los contenidos incluidos en el paquete SCORM y el LMS basado en un modelo de datos predefinido (modelo de datos RTE).

Las 3 sub-especificaciones son utilizadas para definir un paquete SCORM que está formado por una serie de páginas Web. En una de esas páginas está incrustado el VRL junto a una serie de recursos relacionados con los que puede establecer relaciones. En el apartado 4 (Integración Lab-LMS), antes de enumerar los beneficios que aporta esta integración, ya se comentó la idea de presentar el VRL junto a otros elementos que se ha demostrado que pueden aumentar la efectividad del aprendizaje en el VRL cuando se usan de forma conjunta. El formato SCORM permite que estos recursos asociados al VRL, o un subconjunto de los mismos los que decida el diseñador de la experiencia docente, se incluya en el mismo paquete SCORM en el que se incluye el VRL (en la misma página o en otra). De este modo se puede crear una estructura, a la que llamaremos WebLab a partir de ahora, que incluye algo más que el VRL. Esto no significa que no se puedan usar otros recursos pre-WebLab (por ejemplo un test de evaluación inicial y un documento de requisitos técnicos para ejecutar el laboratorio), Post-WebLab (por ejemplo una encuesta para conocer la opinión del alumnado tras ejecutar el laboratorio o un vídeo con la solución correcta a la ejecución del laboratorio) o que se usen mientras utilice el WebLab (por ejemplo un foro para resolución de dudas) que estén implementados en el LMS con sus formatos correspondientes.

SCORM no es un formato muy amigable ni fácil de usar, sobre todo si no se tiene experiencia. Por ello en la UJA se han creado varios modelos de paquetes SCORM que sirven como plantillas para la creación de futuros WebLabs [32] y se han creado una serie de herramientas que facilitan la comunicación entre SCORM y el LMS [31]. La plantilla SCORM de la UJA más utilizada está formada por 4 páginas en las que se aconseja que se incluyan los siguientes recursos:

- Página 1. Información genérica del Laboratorio que trata de definir y enmarcar la experiencia práctica para que los estudiantes entiendan para que sirve, como lo van a realizar y que se van a encontrar.

- Página 2. Contenidos teóricos específicos del VRL y los experimentos que van a realizar posteriormente, incluye recordatorio de la teoría y extensiones de la explicada en clases de teoría más relacionada con los experimentos. Prueba de evaluación para comprobar que el estudiantes posee unos conocimientos mínimos antes de acceder al VRL.

- Página 3. Guiones de prácticas que explican los experimentos a realizar y el propio VRL. 
- Página 4. Prueba de evaluación de competencias/objetivos conseguidos por el alumno tras la ejecución del VRL.

Los primeros WebLabs desarrollados en la UJA utilizaron VRL desarrollados en Java con la herramienta EJS (Easy Java Simulations). Para facilitar la comunicación desde el código Java del VRL hasta el LMS se creó un paquete Java que simplificaba enormemente estas transmisiones en ambos sentidos usando el estándar SCORM. Las herramientas que se han creado para facilitar la comunicación SCORM-LMS. Hoy en día se usa EJsS (Easy Java-JavaScript Simulations) para crear VRL en lenguaje JavaScript y por ello se ha creado una librería JavaScript que facilita las comunicaciones VRL-LMS. Estas comunicaciones son las que hacen que los WebLabs desarrollados en la UJA consigan una integración avanzada, permitiendo realizar aprendizajes adaptados al estudiante. La figura 4 muestra un ejemplo simplificado del esquema del modo de integración utilizado en la UJA con el estándar SCORM.

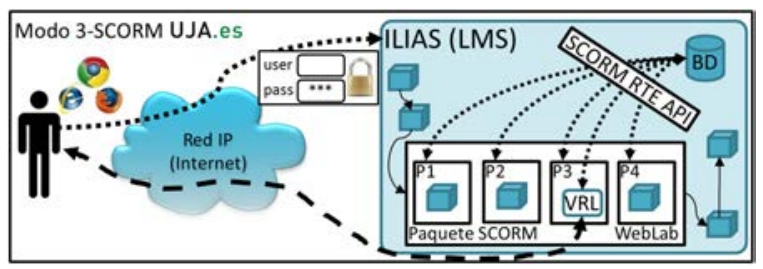

Figura 4. Ejemplo de modo de integración 3 UJA.

\section{CONCLUSIONES}

En este trabajo se ha mostrado un análisis de los modos de integración Laboratorio online-LMS y las ventajas e inconvenientes que se pueden obtener con cada uno de ellos. Cuando la integración es avanzada es posible la comunicación entre el VRL y el LMS, esto permite que el programador de VRL pueda crear experimentos adaptados a los estudiantes y con un aprendizaje más efectivo. Existen tres modos de conseguir la integración avanzada, aunque algunos presentan más problemas que otros. Se ha mostrado el modo elegido por la UJA, basado en alojar el VRL en el propio LMS y conseguir la comunicación mediante el uso de los estándares SCORM y herramientas que han sido desarrolladas por los mismos autores. Un reto que se presenta en el futuro es la estandarización de un vocabulario común para permitir que distintos estándares y desarrollos específicos puedan ser compatibles a la hora de realizar la comunicación VRL-LMS

\section{Agradecimientos}

Este trabajo ha sido parcialmente subvencionado por los proyectos DPI2011-27284, PID30-2014-16 y PI10-AGR-6616.

\section{Referencias}

[1] Abdellaoui N., Gravier C., Belmekki B., and Fayolle J., "Towards the loose coupling between LMS and Remote Laboratories in online engineering education," in IEEE EDUCON 2010 Conference, 2010, pp. 19351940.

[2] Advanced Distributed Learning (ADL), "Content Aggregation Model (CAM) SCORM 2004 4th Ed. v1.1,” 2009.

[3] Advanced Distributed Learning (ADL), "RunTime Environment (RTE) SCORM 2004 4th Ed. v1.1," 2009.

[4] Advanced Distributed Learning (ADL), "Sequencing and Navigation (SN) SCORM 2004 4th Ed. v1.1," 2009.

[5] Advanced Distributed Learning Initiative (ADL). SCORM 2004 4th Ed., 2009. Consultado http://www.adlnet.gov/scorm

$(19 / 06 / 2016)$ :

[6] Bochicchio M. A. and Longo A., "Extending LMS with collaborative remote lab features," Proc. - 10th IEEE Int. Conf. Adv. Learn. Technol. ICALT 2010, pp. 310-314, 2010.

[7] Capterra (2015). "Top LMS Software. All products" Consultado (13/06/2016) en: http://www.capterra.com/learning-managementsystem-software/

[8] de la Torre L., "New Generation Virtual and Remote Laboratories: Integration Into Web Environments 2.0 With Learning Management Systems," SPANISH OPEN UNIVERSITY (UNED), 2013.

[9] Dormido S., "Control learning: present and future,” Annu. Rev. Control, pp. 71-93, 2004.

[10] Ernst E. W., "A New Role for the Undergraduate Engineering Laboratory," IEEE Trans. Educ., vol. 26, no. 2, pp. 49-51, 1983.

[11] Feisel L. D. and Rosa A. J., "The Role of the Laboratory in Undergraduate Engineering Education,” J. Eng. Educ., pp. 121-130, 2005.

[12] Gallardo A., Richter T., Debicki P., Bellido L., Mateos V., and Villagra V., "A rig booking system for on-line laboratories," 2011 IEEE Glob. Eng. Educ. Conf., pp. 643-648, Apr. 2011.

[13] Garcia-Zubia J., Lopez-de-Ipina D., and Orduna P., "Towards a canonical software architecture for multi-device WebLabs," in 31st Annual Conference of IEEE Industrial Electronics Society, 2005. IECON 2005., 2005, p. 6 pp.

[14] Gillet D., Ngoc A., and Rekik Y., "Collaborative web-based experimentation in flexible engineering education," Educ. IEEE Trans., vol. 48, no. 4, pp. 696-704, Nov. 2005.

[15] Gravier C., Fayolle J., Noyel G., Lelevé A., and Benmohamed H., "Distance learning: Closing 
the gap between remote labs and learning management systems," 2006 1st IEEE Int. Conf. E-Learning Ind. Electron. ICELIE, pp. 130-134, 2006.

[16] Gröber S., et al. "Experimenting from a distance-remotely controlled laboratory (RCL)," Eur. J. Phys., vol. 28, no. 3, pp. S127S141, May 2007.

[17] Heradio R., de la Torre L., Galan D., Cabrerizo F. J., Herrera-Viedma E., and Dormido S., "Virtual and remote labs in education: A bibliometric analysis," Comput. Educ., vol. 98, pp. 14-38, Jul. 2016.

[18] Heradio R., De La Torre L., Sanchez J., and Dormido S., "Making EJS applications at the OSP digital library available from Moodle," Proc. 2014 11th Int. Conf. Remote Eng. Virtual Instrumentation, REV. Feb-2014, pp. 112-116.

[19] Hilera J. and Hoya R., Estándares de e-learning: Guía de consulta, vol. 106, no. 493. 2010.

[20] Hoic-Bozic N., Mornar V., and Boticki I., "A Blended Learning Approach to Course Design and Implementation," IEEE Trans. Educ., vol. 52, no. 1, pp. 19-30, Feb. 2009.

[21] IMS QTI, "IMS question \& test interoperability specification v2.0/v2.1", 2006. Consultado $(17 / 06 / 2013)$ http://www.imsglobal.org/question/index.html

[22] IMS LTI "Learning Tools Interoperability". Consultado (17/06/2013) en https://www.imsglobal.org/activity/learningtools-interoperability

[23] Kolberg S., Courivaud D., and Ozbek M. E., "LMS and Interactivity - Technical Issues for Remote Laboratories," in 2007 IEEE 18th International Symposium on Personal, Indoor and Mobile Radio Communications, 2007.

[24] Lonn S., Teasley S. D., and Krumm A. E., "Who needs to do what where?: Using learning management systems on residential vs. commuter campuses," Comput. Educ., vol. 56, no. 3, pp. 642-649, 2011.

[25] Ma J. and Nickerson J. V., "Hands-on, simulated, and remote laboratories," ACM Comput. Surv., vol. 38, no. 3, p. 7-es, 09/2006.

[26] Orduna P. et al. "An Extensible Architecture for the Integration of Remote and Virtual Laboratories in Public Learning Tools," Rev. Iberoam. Tecnol. del Aprendiz., vol. 10, no. 4, pp. 223-233, 2015.

[27] Prendes Espinosa, M. P. (Dir.) (2009). "Plataformas de campus virtual de software libre: Análisis comparativo de la situación actual en las universidades españolas". Informe del Proyecto EA-2008-0257 de la Secretaria de estado de Universidades e Investigación.

[28] Prendes M. P., "Plataformas de campus virtual con herramientas de software libre. Análisis comparativo de la situación actual en las universidades españolas,” 2009.

[29] Ruano I., Gámez J., and Gómez J., "Laboratorio Web SCORM de Control PID con Integración Avanzada," Rev. Iberoam. Automática e Informática Ind. Aceptado (pendiente public.)

[30] Ruano I., Gámez J., Dormido S., and Gómez J., "A Methodology to Obtain Learning Effective Laboratories with Learning Management System Integration," IEEE Trans. Learn. Technol., En proceso de revision (minor).

[31] Ruano I., Gámez J., and Gómez J., "Building SCORM embedded WebLabs with LMS interaction," in 2014 IEEE Frontiers in Education Conference (FIE) Proceedings, 2014.

[32] Ruano I., Gámez J., Dormido S., and Gómez J., "A SCORM based package model for WebLabs," in 2014 IEEE Frontiers in Education Conference (FIE) Proceedings, 2014.

[33] Ruano I., Gómez J., Gámez J., and Estévez E., "Integration of Online Laboratories - LMS via SCORM," in 2013 IEEE International Conference on Systems, Man, and Cybernetics, 2013, pp. 3163-3167.

[34] Rustici Software. Experience API. Consultado (17/06/2013) en https://experienceapi.com/

[35] Sancristobal E. et al., "Integration of internet based labs and open source LMS," Proc. - 3rd Int. Conf. Internet Web Appl. Serv. ICIW 2008, no. Figure 2, pp. 217-222, 2008.

[36] SCOPEO (2011). “Aproximación pedagógica a las plataformas open source en la universidad española”, Marzo de 2011. Monográfico SCOPEO, $\mathrm{n}^{\circ}$ 2. Consultado (13/06/2016) en: http://scopeo.usal.es/wpcontent/uploads/2013/04/scopeom002.pdf

[37] Tawfik M., et al. "Laboratory as a Service (LaaS): A model for developing and implementing remote laboratories as modular components," in 11th International Conference on REV: Remote Engineering and Virtual Instrumentation, 2014, vol. 10, no. 4, pp. 11-20.

[38] Trenas M. A., Ramos J., Gutiérrez E. D., Romero S., and Corbera F., "Use of a new moodle module for improving the teaching of a basic course on computer architecture," IEEE Trans. Educ., vol. 54, no. 2, pp. 222-228, May 2011.

[39] Vargas H., Sánchez J. y Dormido S. (2008). "Proyecto AutomatLabs: Red Interuniversitaria de Laboratorios de Control Automático a través de Internet". XIII Latin American Congress on Automatic Control (CLCA). Mérida (Venezuela), November 2008.

[40] Viedma G., Dancy I. J., and Lundberg K. H., "A web-based linear-systems iLab," in Proceedings of the 2005, American Control Conference, 2005., 2005, pp. 5139-5144. 